\title{
LAMP-2 deficiency leads to hippocampal dysfunction but normal clearance of neuronal substrates of chaperone-mediated autophagy in a mouse model for Danon disease
}

Michelle Rothaug ${ }^{1}$, Stijn Stroobants², Michaela Schweizer ${ }^{3}$, Judith Peters ${ }^{1}$, Friederike Zunke ${ }^{1}$, Mirka Allerding ${ }^{1}$, Rudi D'Hooge ${ }^{2}$, Paul Saftig ${ }^{1}$ and Judith Blanz ${ }^{1 *}$

\begin{abstract}
The Lysosomal Associated Membrane Protein type-2 (LAMP-2) is an abundant lysosomal membrane protein with an important role in immunity, macroautophagy (MA) and chaperone-mediated autophagy (CMA). Mutations within the Lamp2 gene cause Danon disease, an X-linked lysosomal storage disorder characterized by (cardio)myopathy and intellectual dysfunction. The pathological hallmark of this disease is an accumulation of glycogen and autophagic vacuoles in cardiac and skeletal muscle that, along with the myopathy, is also present in LAMP-2-deficient mice. Intellectual dysfunction observed in the human disease suggests a pivotal role of LAMP-2 within brain. LAMP-2A, one specific LAMP-2 isoform, was proposed to be important for the lysosomal degradation of selective proteins involved in neurodegenerative diseases such as Huntington's and Parkinson's disease.

To elucidate the neuronal function of LAMP-2 we analyzed knockout mice for neuropathological changes, MA and steady-state levels of CMA substrates. The absence of LAMP-2 in murine brain led to inflammation and abnormal behavior, including motor deficits and impaired learning. The latter abnormality points to hippocampal dysfunction caused by altered lysosomal activity, distinct accumulation of p62-positive aggregates, autophagic vacuoles and lipid storage within hippocampal neurons and their presynaptic terminals. The absence of LAMP-2 did not apparently affect MA or steady-state levels of selected CMA substrates in brain or neuroblastoma cells under physiological and prolonged starvation conditions.

Our data contribute to the understanding of intellectual dysfunction observed in Danon disease patients and highlight the role of LAMP-2 within the central nervous system, particularly the hippocampus.
\end{abstract}

Keywords: LAMP-2, Danon disease, Mouse model, Lysosome, Chaperone-mediated autophagy, Huntingtin, a-synuclein

\section{Introduction}

The Lysosomal Associated Membrane Protein type-2 (LAMP-2) is a heavily glycosylated protein that, along with LAMP-1, constitutes the majority of all membrane proteins in the lysosome. The carbohydrates of the LAMP proteins form a glycocalyx lining the inner leaflet of the lysosomal membrane that has a compact appearance of only $8 \mathrm{~nm}$ in thickness [1,2]. It is suggested to maintain the structural integrity of the lysosomal

\footnotetext{
* Correspondence: jblanz@biochem.uni-kiel.de

'Institute of Biochemistry, Christian-Albrechts-Universität zu Kiel,

Olshausenstrasse 40, D-24098 Kiel, Germany

Full list of author information is available at the end of the article
}

membrane. However, deglycosylation of the LAMP proteins reduces their stability but does not affect lysosomal integrity and its degradation capacity [3]. LAMP-2, most likely in concert with LAMP-1, has been proposed to contribute to the maturation of autophagic vacuoles $[4,5]$ and phagosomes [6-8] by promoting vesicular fusion events along microtubules [8] and is also involved in endosomal/ lysosomal cholesterol trafficking $[9,10]$.

Alternative splicing of the Lamp 2 gene produces three isoforms, namely LAMP-2A, LAMP-2B and LAMP-2C that are expressed in a tissue specific manner [11-13]. One particular function of the isoform LAMP-2A is to facilitate the selective import and degradation of cytosolic 
proteins in the lysosome via chaperone-mediated autophagy (CMA) [14,15] through recognition of a CMAtargeting motif, a pentapeptide sequence biochemically related to KFERQ present within $25-30 \%$ of all cytosolic proteins [16].

This isoform is also implicated in major histocompatibility complex class II presentation of cytoplasmic antigens [17] as well as in the regulation of T-cell responses [18]. LAMP-2A is also the rate-limiting factor for the neuronal uptake and degradation of aggregation prone proteins via CMA such as $\alpha$-synuclein ( $\alpha$-syn) $[19,20]$ and huntingtin $(\mathrm{Htt})[21,22]$ that are neurotoxic when aggregated [23,24]. Mutations within the Lamp2 gene cause Danon disease, an X-linked "lysosomal glycogen storage disease with normal acid maltase". Danon disease patients suffer from severe skeletal and cardiac myopathy as well as intellectual dysfunction [25-27]. Interestingly, one Danon disease patient was identified to carry a mutation only affecting LAMP-2B, underlining the importance of this specific isoform [26].

In addition to increased mortality up to post-natal day 40 and reduced size, we have previously described (cardio)myopathy and the pronounced accumulation of autophagic vesicles in cardiac and skeletal muscle of LAMP-2 knockout mice $[4,28]$ similar to those reported in human patients [26]. Neuropathological changes in post-mortem material from a Danon disease patient have been observed [29] which warranted a more in-depth analysis of LAMP-2-deficient murine brain for the presence of neuropathological signs.

Here, we report that absence of LAMP-2 in mice leads to inflammatory changes and lysosomal accumulation of electron dense material within neurons of the central nervous system (CNS). Behavioral abnormalities such as impaired memory point to hippocampal dysfunction caused by perturbed lysosomal activity, accumulation of p62-positive aggregates as well as cholesterol storage within neurons of the hippocampus. In addition, hippocampal neurons displayed a distinct accumulation of lipofuscin and autophagic vacuoles containing amorphous and multilamellar material. Despite its proposed role for the lysosomal degradation of $\alpha$-syn, $\mathrm{Htt}$, the myocytespecific enhancer factor-2D (MEF2D) and glyceraldehyde3-phosphate dehydrogenase (GAPDH) in lysosomes via CMA, steady-state levels of these proteins were unchanged in LAMP-2-deficient brain tissue and in neuroblastoma cells where LAMP-2 was stably down-regulated. Our data demonstrate an essential role of the LAMP-2 protein within the CNS for cognitive functions and autophagy.

\section{Materials and methods}

Experimental animals

LAMP-2-deficient mice were described previously $[4,30]$. Only male animals were used in this study due to the
X-chromosomal location of the Lamp2 gene. Therefore, LAMP-2-deficient animals that were backcrossed into C57/BL6-N (Charles River) are referred to as LAMP$2^{-/ y}$. Animals were maintained in a conventional animal facility. All procedures performed in this study involving animals were in accordance with the ethical standards set by the National Animal Care Committee of Germany.

\section{Materials}

Substrates for activity assays (p-nitrophenyl- $\alpha$-D-glucuronide and $\mathrm{p}$-nitrophenyl- $\mathrm{N}$-acetyl- $\beta$ - $\mathrm{D}$-glucosiminide) and filipin complex from Streptomyces filipinensis were purchased from Sigma Aldrich (Steinheim, Germany). Reagents for molecular biology and protein standards were obtained from Fermentas (St. Leon-Rot, Germany). Chemicals for buffers and RNA isolation were from Roth (Karlsruhe, Germany) and the BCA protein assay kit and Western blotting reagents from Pierce (Rockford, USA) and Amersham (Little Chalfont, United Kingdom), respectively. Complete ${ }^{\odot}$ protease inhibitor and PefaBloc ${ }^{\circ}$ were purchased from Roche (Mannheim, Germany). Media for common cell culture was acquired from PAA (Pasching, Austria).

\section{(Immuno)histology, filipin staining and electron microscopy}

For antibody labelling, mice were perfused transcardially with $4 \%$ paraformaldehyde (PFA) in $0.1 \mathrm{M}$ phosphate buffer (PB). The brains were dissected and post-fixed for 4 hours. Thereafter, they were embedded in paraffin or incubated in $30 \%$ sucrose $/ 0.1 \mathrm{M}$ PB. Five $\mu \mathrm{m}$ paraffin sections or $35 \mu \mathrm{m}$ free-floating cryosections were cut. 3,3'-Diaminobenzidine (DAB) staining was carried out on free-floating cryosections using the $\mathrm{ABC}$ kit followed by the Elite $\mathrm{DAB}$ staining kit according to the manufacturer's instructions (Vector Laboratories, Enzo Life Sciences, Lörrach, Germany). Periodic-Acid-Schiff (PAS) staining was carried out according to common lab protocols. TUNEL staining was performed using the ApopTag ${ }^{\circ}$ peroxidase in situ apoptosis detection kit (Millipore, Schwalbach, Germany) according to the manufacturer's instructions. Sections were mounted in Eukitt (Sigma Aldrich, Steinheim, Germany) and visualized using a BX50 microscope (Olympus, Hamburg, Germany). Filipin histochemistry was performed on $35 \mu \mathrm{m}$ vibratome sections at room temperature. Sections were washed $2 \times 10$ minutes in phosphate buffered saline (PBS), $2 \times 10$ minutes in $0.2 \%$ saponin/PBS and then incubated in filipin $(0.05 \mathrm{mg} / \mathrm{ml})$ for 20 minutes. After washing $2 \times 10$ minutes in $0.02 \%$ saponin/PBS and $2 \times 10$ minutes in PBS, sections were mounted in Prolong anti-fade mounting solution (Invitrogen, Darmstadt, Germany) and stored at $4^{\circ} \mathrm{C}$. For electron microscopy (EM) mice were perfused and post-fixed with 
4\% PFA/1\% glutaraldehyde in $0.1 \mathrm{M} \mathrm{PB}$. Brains were dissected and stored in fresh EM fixative at $4^{\circ} \mathrm{C}$ until further treatment. Following osmication with $1 \%$ osmiumtetroxide in cacodylate buffer the sections were dehydrated using ascending alcohol concentration steps, followed by two rinses in propylene oxide. Infiltration of the embedding medium was performed by immersing the pieces in a 1:1 mixture of propylene oxide and Epon (Carl Roth GmbH \& Co. KG, Karlsruhe, Germany) and finally in neat Epon before hardening at $60^{\circ} \mathrm{C}$ for 48 hours. Semithin sections $(0.5 \mu \mathrm{m})$ were prepared for light microscopy, mounted on glass slides and stained for 1 minute with $1 \%$ toluidine blue. Ultrathin sections $(60 \mathrm{~nm})$ were cut and mounted on copper grids. Sections were stained using uranyl acetate and lead citrate. Thin sections were examined and photographed using an EM902 (Zeiss, Jena Germany) electron microscope equipped with a Megaview III digital camera (Albert Tröndle, Moorenweis, Germany).

\section{LAMP-2 knockdown in N2a cells}

Oligonucleotides were purchased from Sigma Aldrich (Steinheim, Germany) and siRNA sequences were determined using the DSIR program (http://biodev.extra.cea. fr/DSIR/DSIR.html). The corresponding scramble RNA sequence was created using siRNA wizard (www.sirnawizard. com/scrambled.php). Oligonucleotide sequences used were as follows: forward primer LAMP-2 shRNA 5'-3' [gatcccc GGAGATGAATTTCACAATAttcaagagaTATGTGAAAT TCATCTCCttttta], reverse primer LAMP-2 shRNA 5'-3' [agcttaaaaaGGAGATGAATTTCACAATAtctcttgaaTAT TGTGAAATTCATCTCCggg], forward primer scmbl shRNA 5'-3' [gatccccGATTGCTAAGACAAGTAAttcaaga gaTTACTTGTCTATAGCAATCttttta], reverse primer scmbl shRNA 5'-3' [agcttaaaaaGATTGCTATAGACAAG TAAtctcttgaaTTACTTGTCTATAGCAATCggg]. Short hairpin sequences were transferred between the BglII und HindIII sites in the pSUPER vector (OligoEngine, Seattle, USA) according to the manufacturer's instructions. Mouse neuroblastoma cells (N2a) were transfected using polyethylenimine [31]. Stable transfection with G418 (PAA, Pasching, Austria) was initiated 48 hours post-transfection at a concentration of $100 \mu \mathrm{g} / \mathrm{ml}$ and increased by $100 \mu \mathrm{g} /$ $\mathrm{ml}$ every 48 hours until a maximum of $400 \mu \mathrm{g} / \mathrm{ml}$ G418 was reached. Cells were maintained for at least 2 weeks in stable media before preparation for immunoblotting. Cells were harvested in ice cold PBS with protease inhibitors (complete ${ }^{\circ}$ from Roche, Mannheim, Germany). Messenger RNA was isolated from HeLa cells using the NucleoSpin ${ }^{\circ}$ RNAII kit (Macherey-Nagel, Düren, Germany) according to the manufacturer's instructions. Subsequently, LAMP-2A was cloned in to the pcDNA3.1 Hygro $^{+}$vector (Invitrogen, Darmstadt, Germany) from cDNA transcribed using the RevertAid cDNA synthesis Kit (Fermentas, St. Leon-Rot, Germany).

\section{Behavioral study}

LAMP-2 knockout mice and wild-type controls (both: $\mathrm{n}=4$ ) were examined in a battery of behavioral tests to assess functional consequences of LAMP-2 deficiency. Different tests of motor performance were included. Home cage activity was analyzed in $20 \mathrm{~cm}$ vs $30 \mathrm{~cm}$ transparent cages which were placed between 3 infrared beams. Total number of beam crossings was recorded for 23 hours during 30 minute intervals (start $4 \mathrm{pm}$ ). Grip strength was measured using a T-shaped bar connected to a digital dynamometer (Ugo Basile, Comerio, Italy). Mice were placed in such a way that they grabbed the bar spontaneously and were softly pulled backwards by the tail until they released their grip. Ten such readouts were recorded. Motor coordination and equilibrium were tested using an accelerating rotarod (MED Associates Inc., St. Albans, Vermont, USA). Mice were first trained to maintain balance for 2 minutes at a constant speed of $4 \mathrm{rpm}$. This training trial was followed by four test trials, during which the rod accelerated from 4 to $40 \mathrm{rpm}$ in 5 minutes. Consecutive trials were separated by a 10 minute intertrial interval. Latency to falling off the rod was recorded up to 5 minutes. In the swim test, mice were placed in a circular pool (diameter $150 \mathrm{~cm}$; water temperature $26^{\circ} \mathrm{C}$ ) for 30 seconds. The animals were tracked using Ethovision tracking equipment and software (Noldus Bv, Wageningen, The Netherlands) for assessment of swimming velocity. Gait analysis was performed while mice were ventrally video-tracked on a transparent treadmill belt (Digigait, MouseSpecifics Inc, MA, USA). Following brief habituation to the apparatus, mice were tested at a constant belt speed of $16 \mathrm{~cm} / \mathrm{s}$. Different parameters were extracted from these video data using Digigait analysis software: base-widths (distance between contralateral paws), stride lengths (distance between subsequent placements of the same paw) and maximal paw area (peak stance). As an indicator of emotional function, an exploration test was performed in a plexiglass arena $(42 \mathrm{~cm} \times 26 \mathrm{~cm} \times 30 \mathrm{~cm})$ which mice could freely explore for 5 minutes. Mice were video-tracked with ANY-maze ${ }^{\mathrm{Tw}}$ Video Tracking System software (Stoelting Co., IL, USA). Total distance travelled was calculated as a measure of exploratory activity. Finally, mice completed different learning and memory tasks. Working memory was assessed in a Y-maze consisting of 3 arms $(5 \mathrm{~cm}$ wide, $30 \mathrm{~cm}$ long and enclosed by $30 \mathrm{~cm}$ high wall made of grey plastic) [32]. Mice were placed in the center for 10 minutes exploration of all arms. Locomotion was observed by a webcam connected to a screen. Entries into all arms were noted and an alternation was counted if an animal entered three different arms consecutively. Percentage spontaneous alternation was calculated according to following formula: [(number of alternations)/(total number of arm entries -2$)]^{*} 100$. Contextual and cued fear conditioning 
was evaluated in a plexiglass test chamber $(26 \times 22 \times$ $18 \mathrm{~cm}$ high), containing a grid floor to deliver an electric shock using a constant current shocker (MED Associates Inc., St. Albans, Vermont, USA). The test set-up was placed inside a sound attenuated chamber. On the first day of the experiment, animals were placed in the testing chamber and allowed to acclimate for 5 minutes. On the second day, animals were again placed in the testing chamber and after 2 minutes of exploration (baseline score), a buzzer was sounded for 30 seconds. This auditory stimulus, the conditional stimulus, was followed by a 2 second foot shock $(0.3 \mathrm{~mA})$, the unconditional stimulus. After the shock, mice were allowed to explore once more for 1 minute before they received a second conditionalunconditional stimulus pairing. Finally, they were allowed to explore for another minute. Twenty-four hours later, on the third and last day, the animals were placed in the same context for 5 minutes exploration (contextual fear assessment). After 90 minutes, the mouse was again placed in the test chamber. Environmental and contextual cues were changed: a white plate was placed on the grid, light was switched on and minute extract was used to alter the smell. After 3 minutes of free exploration (pre-cue phase), the auditory stimulus was delivered for 3 minutes (cued fear assessment). Freezing behavior was recorded every 10 seconds during each trial block using the standard interval sampling procedure.

\section{Antibodies}

Goat-Alexa Fluor 488 and 594 conjugated anti-mouse or anti-rabbit secondary antibodies were used (Molecular Probes, Eugene, USA). Primary antibodies included rat-anti-CD68 (Serotec, Edinburgh, UK), rabbit-anti-actin, mouse-anti-GFAP, mouse-anti-MAP-2 (Sigma Aldrich, Steinheim, Germany), mouse-anti-huntingtin (Millipore, Schwalbach, Germany), rabbit-anti-LC3 and rabbit-antip62 (Enzo Life Sciences, Lörrach, Germany), rat-antiLAMP-2 (Ab193) and rat-anti-LAMP-1 (1D4B) (DSHB, Iowa City, US), rabbit-anti-LAMP-2A (Pineda, Berlin, Germany), rabbit-anti-cathepsin D (a kind gift from Prof. J. Aerts), mouse-anti-MEF2D (BD Biosciences (Heidelberg, Germany), rabbit-anti-GAPDH and rabbit-anti- $\alpha$-synuclein (C-20) (Santa Cruz, Dallas, US), rabbit-anti-caspase-3, rabbit-anti-phospho-PRAS40 and rabbit-anti-PRAS40 (Cell Signalling, Frankfurt am Main, Germany) and rabbit-antiNSE (Abcam, Cambridge, UK).

\section{SDS-PAGE, Immunoblotting, assays monitoring MA and CMA substrate degradation and enzyme activity}

For starvation assays, cells were extensively washed and cultured for 3 or 24 hours in Earl's Balanced Salt Solution (EBSS) or Dulbecco's Modified Eagle's Medium (DMEM) without serum, supplemented with antibiotics before harvesting. Enzyme activity assays were performed on lysates from 54 -week-old mice ( $\mathrm{n}=3$ wild-type and $\mathrm{n}=3$ LAMP-2-deficient) as previously described [33]. N2a cells and brain tissue were lysed in RIPA buffer and denatured in Laemmli. For immunoblotting, protein samples were loaded on an SDS gel and blotted on a nitrocellulose or PVDF membrane (Roth, Karlsruhe, Germany). Membranes were probed with the relevant primary and secondary antibodies. Peroxidase-conjugated secondary antibodies were detected by chemiluminescence (SuperSignalWest, Pierce, Pittsburgh, USA). Densitometric analyses were performed with Image J software (http:// imagej.nih.gov/ij/). Samples were normalized to the loading control and are presented as protein levels relative to wild-type or untreated, non-transfected samples where appropriate.

\section{Preparation and maintenance of primary neuronal and astroglial culture}

Primary neurons were prepared as described previously [34] from the cortices of gestational day 15 embryos. After 3 days, cultured cells were treated with $10 \mu \mathrm{M}$ cytosine arabinoside. Primary glial cultures were prepared as above but cultured in minimum essential medium with $10 \%$ horse serum and penicillin/streptomycin. Cells were fixed in $4 \%$ paraformaldehyde diluted in PBS after 14 days in vitro.

\section{Relative quantitative RT-PCR}

Messenger RNA was isolated from whole brain using the NucleoSpin ${ }^{\circ}$ RNAII kit (Macherey-Nagel, Düren, Germany) according to the manufacturer's instructions. Complementary DNA was created using $2 \mu \mathrm{g}$ total RNA and amplified using oligodT primers and the RevertAid cDNA synthesis Kit (Fermentas, St. Leon-Rot, Germany). Negative controls (RT-) were carried out in the absence of reverse transcriptase to check for genomic contamination. Quantitative RT-PCR, using actin as the house-keeping gene, was carried out using the Universal ${ }^{\circ}$ probe library and Lightcycler 480 II from Roche (Mannheim, Germany) according to the manufacturer's instructions. Relative expression was calculated using an efficiency-corrected comparative quantitation method [35]. To determine efficiency of each primer set, a standard curve was plotted using 10-fold serial dilutions of a mix of applied cDNA, followed by a logarithmic conversion and a linear response generated.

\section{Statistical analysis}

All values are expressed as the mean \pm standard error of the mean. Differences among mean values were analyzed via a two-tailed, unpaired Student $t$-test using Microsoft Excel software or one-way ANOVA followed by Tukey post-Hoc test, using GraphPad Prism 5, where multiple samples were compared. Behavioral data were analyzed 
with SigmaStat software using Student $t$-test MannWhitney Rank Sum Test or Repeated-Measures ANOVA where appropriate. The null hypothesis was rejected at $\mathrm{p}<0.05$.

\section{Results \\ Loss of LAMP-2 expression causes neuropathological changes in mice}

Similarities in the pathological presentation of Danon disease in cardiac and skeletal muscle of humans and LAMP-2-deficient (LAMP- $2^{-/ y}$ ) mice $[4,28]$ that were of a mixed genetic background (SVJ-129/C57BL6-J (Harlan)) highlight the validity of this mouse as a model for the human disease. The neuropathological characterization of LAMP-2-deficient mice was carried out in animals backcrossed into C57/BL6-N (Charles River). We recently reported that backcrossing of LIMP-2 (Lysosomal Integral Membrane Protein type-2) knockout mice, that were maintained within the same mixed genetic background as the LAMP-2-deficient mice, into C57/BL6-N led to a deterioration of their phenotype including accumulation of $\alpha$-syn, severe CNS impairments and premature death [36]. In contrast, LAMP-2-deficient mice of the mixed and C57/BL6-N background were macroscopically indistinguishable from each other.

Histological analysis of the CNS of 12-month-old LAMP-2-deficient mice revealed wide-spread astrogliosis within all brain regions, including the hippocampus, as illustrated by immunohistochemistry (Figure 1a) and immunoblotting (Figure 1b) with an antibody directed against the Glial Fibrillary Acidic Protein (GFAP). Mild microgliosis (Figure 1c and Additional file 1a), as depicted by immunohistochemical staining using an antibody specific for the microglia resident protein macrosialin/CD68, was observed throughout LAMP-2-deficient brains. In the subiculum of the hippocampus and pons, microgliosis was most prominent. Here, microglia assumed a morphology indicative of their activation (Figure 1c, left and middle panels). Toluidine blue stained sections of LAMP-2deficient brains, revealed darkly stained degenerating neurons within the hippocampus, particularly the subiculum and the CA3 region (Additional file 1b). However, no indication of apoptosis was evident, as highlighted by Terminal deoxynucleotidyl transferase dUTP Nick End Labeling (TUNEL) (Additional file 1c) and lack of caspase-3 cleavage (Additional file 1d). Using an antibody (Abl93, Development Studies Hybridoma Bank) that recognizes all LAMP-2 isoforms (pan LAMP-2), LAMP-2 was shown to be expressed in both primary cultured neurons (Figure 2a) and astrocytes (Figure 2b). Immunoblot analysis of different brain regions, obtained from wild-type mice, revealed ubiquitous expression of LAMP-2 within the CNS. Quantification of LAMP-2 immunoblot levels relative to cortex revealed the highest expression of
LAMP-2 in the cerebellum and the lowest in the hippocampus (Figure 2c). Expression of both LAMP-2A and LAMP-2B isoforms in the hippocampus was also evident by quantitative (q) RT-PCR (Figure 2d/e). LAMP-2A appeared to be the most abundant isoform expressed in brain (Figure 2d) whereas transcripts of LAMP-2C, an isoform implicated in the uptake of RNA and DNA [37] was not detectable in brain using semi-quantitative PCR (data not shown). Immunohistochemistry of brain sections showed localized enriched expression of LAMP-2 in neurons especially in pyramidal neurons of the hippocampus (Figure 2f, upper panels and Additional file 2a, upper panels) and in the pons (Figure 2f, middle panels and Additional file 2a, lower panels). Despite its similar distribution, we did not observe a compensatory upregulation of LAMP-1 in LAMP-2-deficient brain as illustrated by LAMP-1 immunoblot (Additional file 2b) and immunohistological (Additional file 2c) analysis. From our data we conclude that LAMP-2 is an abundant protein of neuronal tissue and that loss of its expression causes neuroinflammation in mice.

\section{Behavioral anomalies as a result of LAMP-2 deficiency}

Danon disease patients present with neurological manifestations in the form of intellectual dysfunction [25-27]. LAMP-2-deficient mice were subjected to a battery of behavioral tests that included measures of motor performance (gait analysis, grip strength, activity, rotarod and swim test) and exploration as well as indices of learning and memory (Y-maze, contextual fear conditioning). Gross motor behavior appeared unaltered in LAMP-2-deficient mice, as there were no significant differences when compared to wild-type controls in grip strength, rotarod performance and home cage activity (Additional file 3a-c). Likewise, rudimentary gait parameters such as base-widths and stride lengths were similar between genotypes (data not shown). However, maximal paw area was reduced in LAMP-2-deficient mice (Figure 3a). Although this might indicate altered plantar placing in the context of a motor deficit, it is more likely a consequence of reduced body weight in these mice (data not shown). Impaired motor performance of LAMP-2deficient mice was evident in the swim test exemplified by severely reduced swimming velocity (Figure $3 \mathrm{~b}$ ). This effect was not confirmed to be caused by muscular weakness as grip strength performance was normal. Reduced exploratory activity was observed in the exploration test (Figure 3c). Considering unaltered levels of home cage activity (Additional file 3c), this effect does not appear to reflect general hypolocomotion of LAMP-2-deficient mice. Rather, it could indicate blunted affective response to the novel environment. LAMP-2-deficient mice show an increased freezing percentage during the habituation phase 


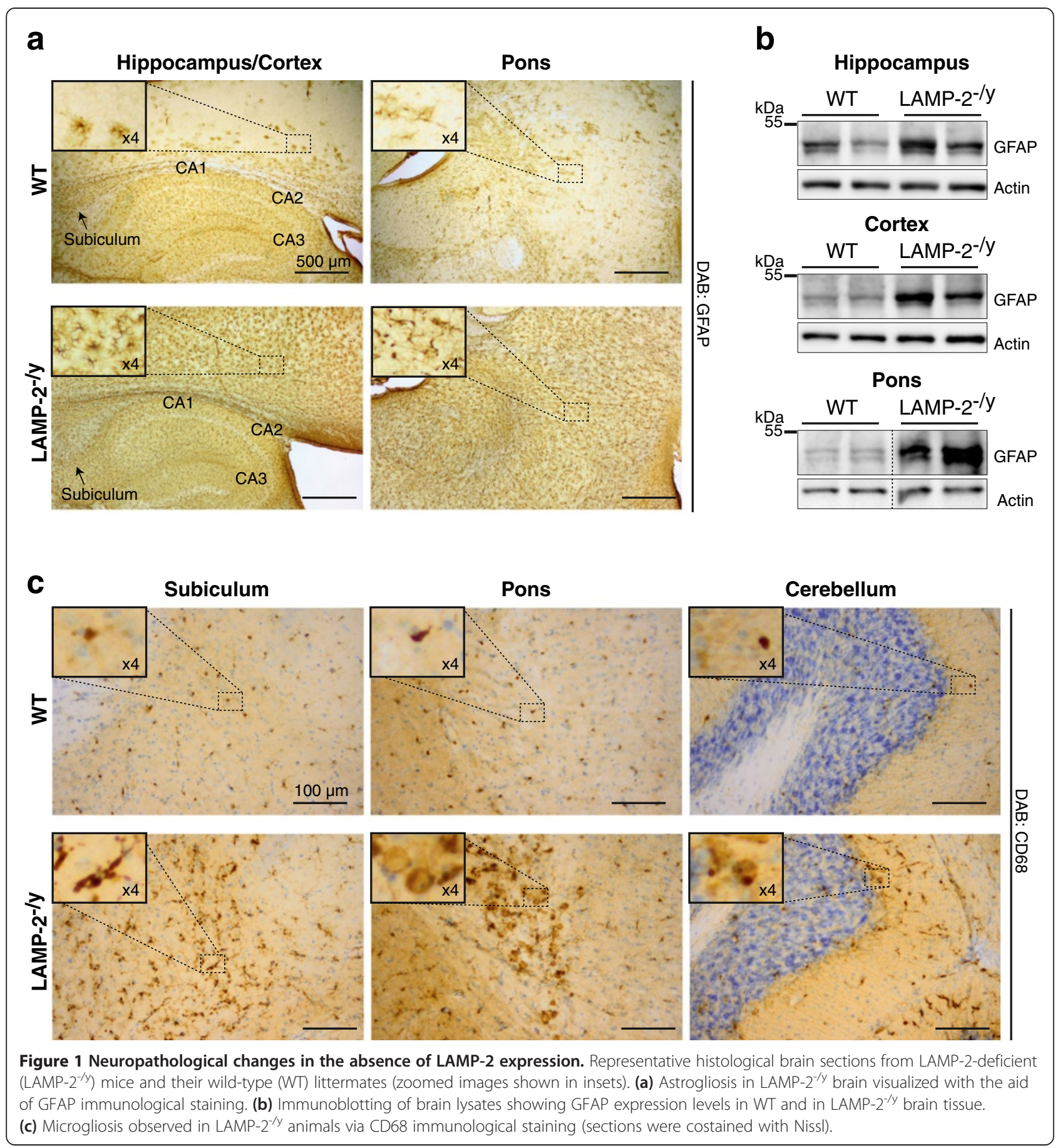

of the contextual fear conditioning experiment, which may be the consequence of reduced exploratory movement. However, fear conditioning as well as contextual and cued fear memory was intact in LAMP-2-deficient mice (Figure 3d). In the Y-maze task, LAMP-2-deficient mice made relatively less alternations (Figure $3 \mathrm{e}$ ) in comparison with wild-type control mice, indicating impaired working memory. In conclusion, LAMP-2-deficient mice show particular motor impairments, reduced exploratory activity and signs of impaired memory, consistent with hippocampal dysfunction.

\section{Lysosomal dysfunction in hippocampal neurons}

The presence of neuroinflammation and behavioral anomalies in mice lacking LAMP-2 expression highlights the importance of this lysosomal protein in brain. To assess whether loss of LAMP-2 leads to lysosomal dysfunction that correlates with the observed neuropathological 

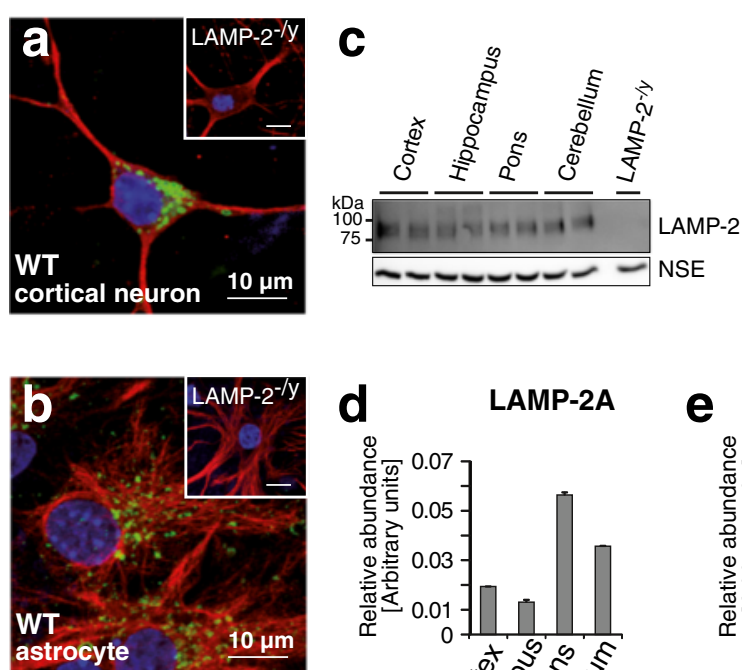

d

LAMP-2A
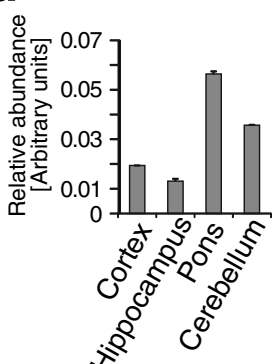

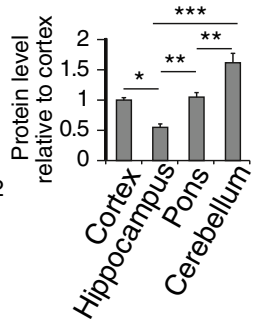

e

LAMP-2B

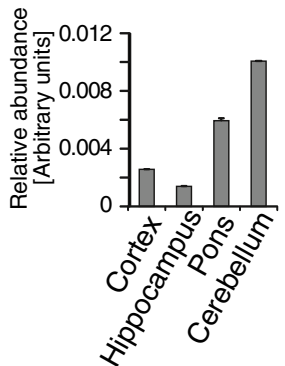

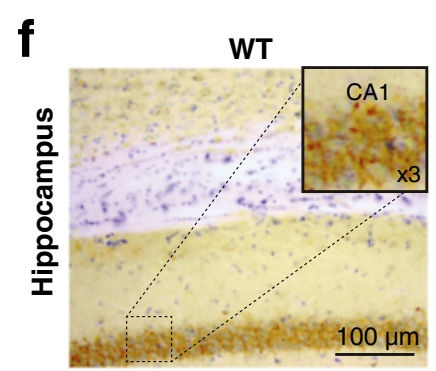
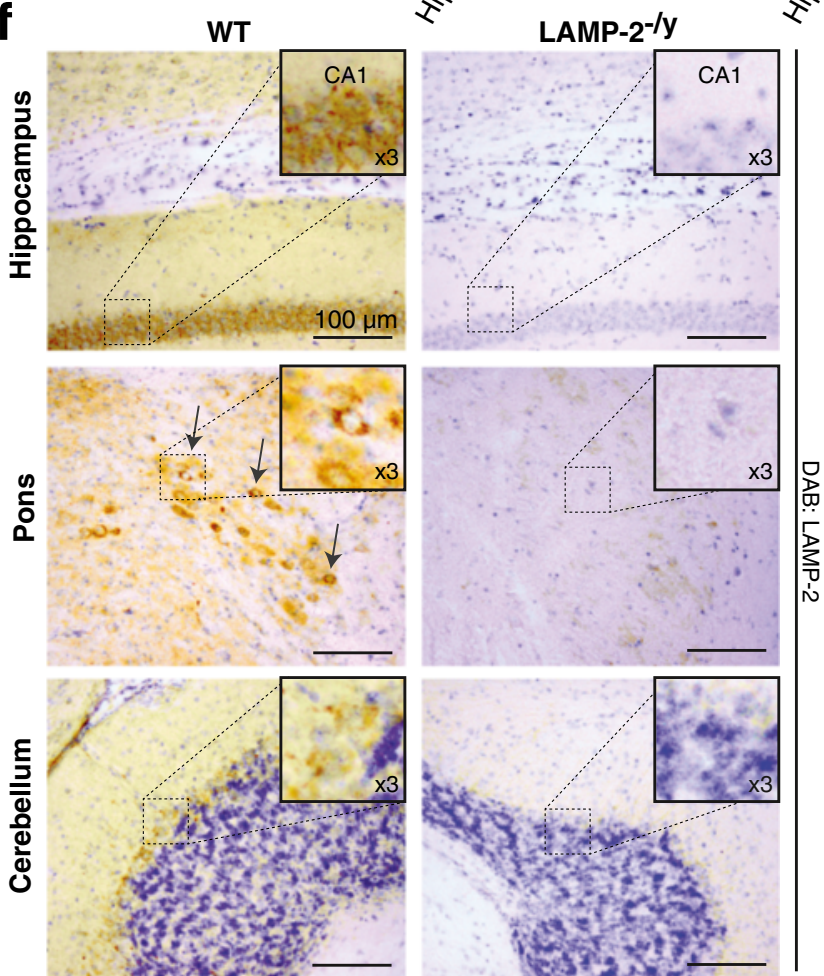

Figure 2 LAMP-2 expression in brain. LAMP-2 (green) vesicular staining detected in (a) MAP-2 (red) positive cultured primary neurons and (b) GFAP (red) positive primary astrocytes (LAMP-2-deficient (LAMP-2-1y) cultures were used to control for antibody specificity). (c) Immunoblot and respective densitometric analysis of LAMP-2 $(n=5)$ showing its expression throughout murine brain (cortex from a LAMP- $2^{-1 y}$ mouse was used to show specificity of the LAMP-2 antibody; actin was used as a loading control; ${ }^{*} p<0.05,{ }^{* *} p<0.01$, ${ }^{* *} p<0.001$ ). Quantitative RT-PCR of isoforms (d) LAMP-2A and (e) LAMP-2B in respective brain regions. (f) Representative histological brain sections stained for LAMP-2 using the DAB method and counterstained with Nissl. No signal was detected in LAMP-2 ${ }^{-1 y}$ brains. Enriched expression was observed in the pyramidal layer of the hippocampus, within the Purkinje cell layer of the cerebellum and within a region of the pons. Insets show zoomed images of regions outlined.

abnormalities, we determined enzyme activities of the lysosomal hydrolases $\beta$-hexosaminidase and $\beta$-glucuronidase as well as the maturation of cathepsin $\mathrm{D}$ as a means of evaluating lysosomal function. Whereas a significant increase of all forms of cathepsin D (pro (p), immature (i) and mature $(\mathrm{m})$ ) was observed in the hippocampus and in the cortex (Figure 4a, Additional file 4), no marked change in the maturation of the enzyme was detected (Figure 4a). 
a

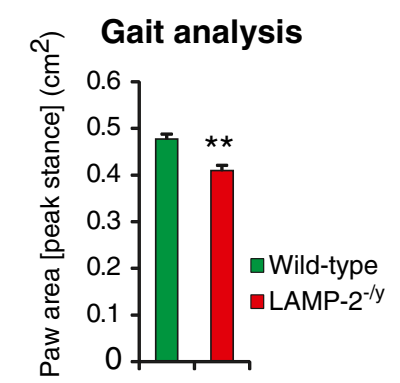

d

Contextual fear conditioning

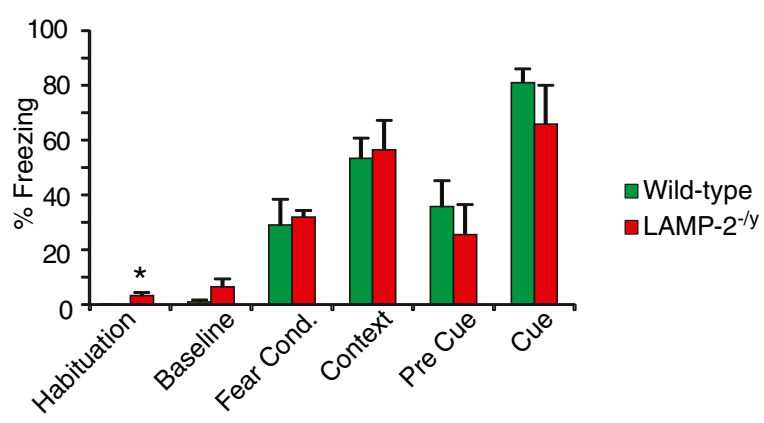

C

\section{Exploration}

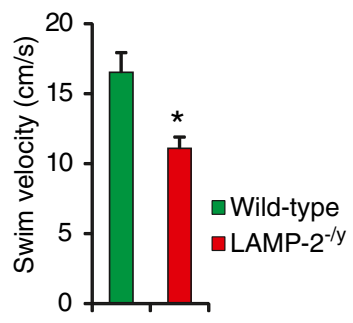

e

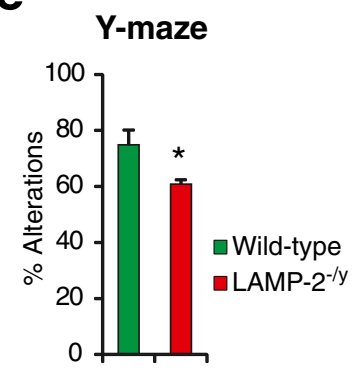

Figure 3 Abnormal behavior as a result of LAMP-2 deficiency in mice. (a) LAMP-2-deficient (LAMP-2/y) mice show a significantly reduced paw area at the peak of stance in comparison with wild-type mice. (b) LAMP-2-1y mice swim significantly slower than wild-type controls. (c) Path length during exploration of a novel environment is significantly reduced in LAMP-2-1y mice. (d) Freezing percentage is significantly increased in LAMP-2-1y mice during the habituation phase. There was no difference in fear conditioning and contextual or cued fear memory. (e) Y-maze working memory: LAMP-2-1/y mice make significantly less spontaneous alternations $\left({ }^{*} \mathrm{p}<0.05 ;{ }^{* *} \mathrm{p}<0.01\right)$.

Additionally, the hippocampus of LAMP-2 knockout mice displayed a significant increase in $\beta$-hexosaminidase (Figure $4 \mathrm{~b}$ ) and $\beta$-glucuronidase (Figure 4c) activity whereas in the pons and cerebellum only $\beta$-hexosaminidase activity was elevated. In summary, among all other brain regions investigated, the hippocampus of LAMP-2deficient mice showed the most consistent signs of alterations in lysosomal enzyme activity.

\section{Lipid storage and autophagic vacuoles in LAMP-2-deficient hippocampal neurons}

Degradation of long-lived proteins which has been shown to be impaired in LAMP-2-deficient hepatocytes [4] mainly occurs by autophagy [38]. The detection of increased levels of proteins that are pivotal for the autophagic flow and are degraded in the lysosome, such as the adaptor protein sequestosome 1 (SQSTM1/p62) [39,40], is widely used as an indication of impaired autophagy. Immunohistochemical analysis of LAMP-2-deficient brain revealed a distinct accumulation of p62-positive aggregates specifically within the subiculum and CA1 region of the hippocampus and the pons (Figure 5a).
Periodic-Acid-Schiff (PAS) staining identified carbohydrate-conjugates within the brain of one Danon disease patient [29]. In neuronal tissues of LAMP-2-deficient mice, no apparent PAS-positive storage was evident (Additional file 5a). Filipin staining displayed storage of free cholesterol exclusively in the subiculum of LAMP-2deficient mice (Figure 5b). High power electron micrographs revealed the presence of Lipid Whorls (LW) that are implicated in lysosomal storage disease and cholesterol accumulation [41] in degenerating neurons within the CA3 and subiculum of LAMP-2-deficient mice (Figure 5c, upper left and lower right panels). Additionally, Lipofuscin (L) and Autophagic Vacuoles (AVs) containing amorphous or multilamellar material were evident within dystrophic neurites and axonal spheroids in the hippocampus (Figure 5c) which were not present in control mice (Additional file 5b). Storage of AVs within axons led to disruption of the presynaptic terminal highlighted by complete absence of synaptic vesicles (Figure $5 \mathrm{c}$, bottom left panel). Storage was observed both within the soma and neurites of cells. Our histological data indicate that loss of LAMP-2 expression causes lysosomal/autophagic 


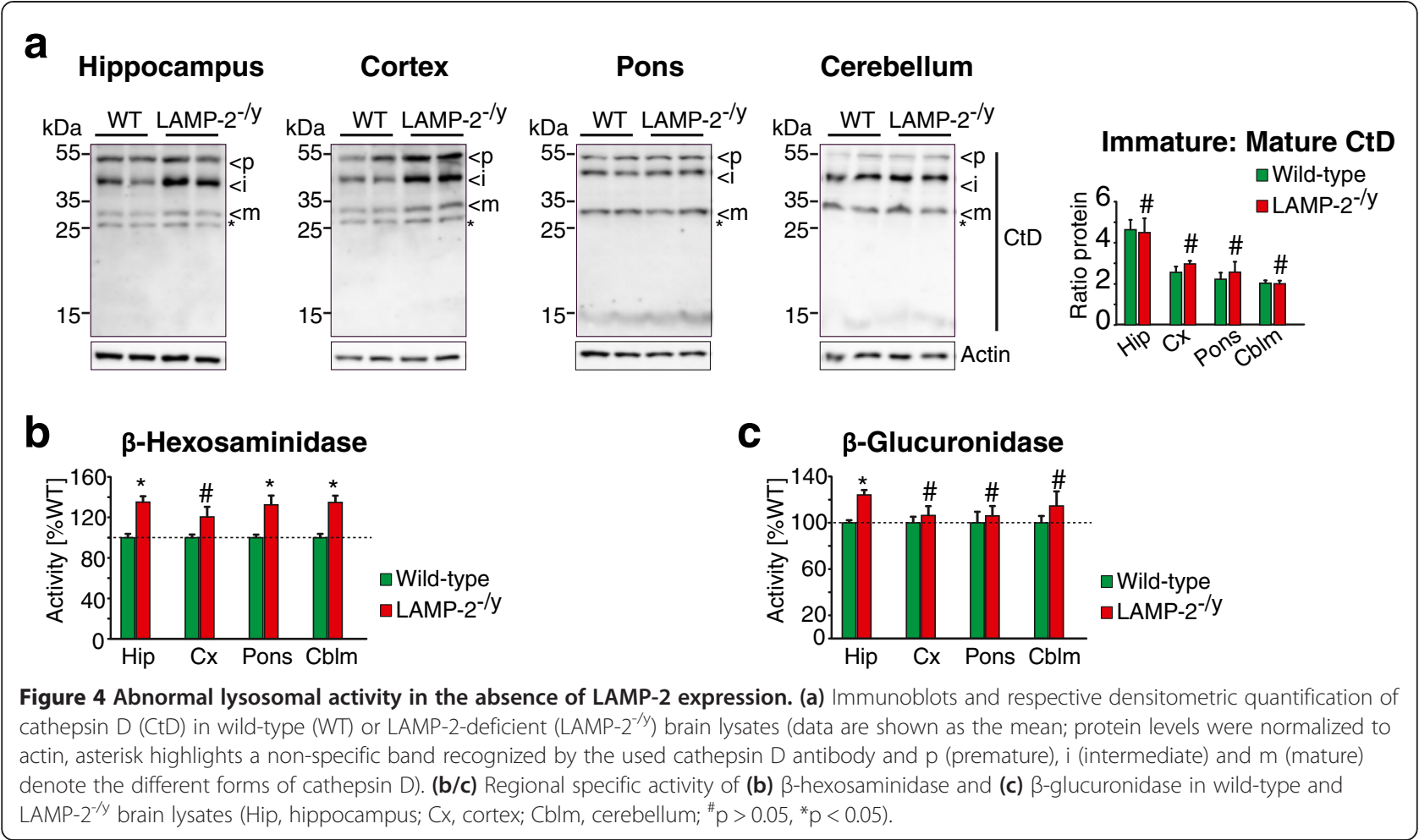

disturbances particularly in the hippocampus of mice. However, in hippocampal extracts that were prepared from LAMP-2 knockout mice we found no significantly increased levels of LC3-II or p62 (Additional file 5c). The ratio of phosphorylated proline-rich Akt substrate 40 (PRAS40) which is known to negatively regulate autophagy [42,43] was also not changed in LAMP-2-deficient samples (Additional file $5 \mathrm{c}$, middle panel) indicating no overall increase in basal MA when LAMP-2 is absent. Proteasomal activity was also unaltered since levels of poly-ubiquitinated proteins were unchanged in LAMP-2 knockout brain samples when compared to wild-type controls (data not shown).

Loss of LAMP-2 does not influence the steady-state levels of CMA substrates and is dispensable for starvation induced clearance of these proteins

LAMP-2A is reported to be the rate-limiting factor in CMA $[14,15]$. Such proteins that are degraded via CMA include those associated with neurodegenerative diseases such as MEF2D, Htt as well as the glycolytic enzyme GAPDH [15]. Thus, we assessed a potential accumulation of these substrates, due to the lack of the CMA receptor LAMP-2A, through analysis of brain lysates from knockout animals as well as in vitro after stable knockdown of LAMP-2 in murine neuroblastoma cells (N2a). Absence of LAMP-2 expression in cortex and hippocampus (Figure 6a) did not affect the steady-state levels of MEF2D (Figure 6b), GAPDH (Figure 6c) or $\mathrm{Htt}$
(Figure 6d, Additional file 6a). Expression levels of Htt were also found to be unchanged in striatum (Figure $6 \mathrm{~d}$ ) which is the primary site of neuronal damage in Huntington`s disease. Similarly, stable knockdown of LAMP2 in N2a cells using shRNA directed against LAMP-2 mRNA significantly reduced protein levels of LAMP-2 (Figure 6e) but not LAMP-1 (Additional file 6b) and did not alter steady-state levels of MEF2D (Figure 6f), GAPDH (Figure 6g) or Htt (Figure 6h). Furthermore, an accumulation of MEF2D within the cytosol, which has been reported after blockage of CMA by LAMP-2 silencing [44], was not revealed neither in LAMP-2-deficient primary neurons (Additional file 6c) nor in brain sections (Additional file $6 \mathrm{~d}$ ). The typical nuclear localization of this protein was observed independent of the expression of LAMP-2.

Prolonged starvation of cells for 24 hours in serum-free media is known to induce CMA [45]. In order to investigate LAMP-2-dependent clearance of substrates after extended starvation, N2a cells, stably transfected with either LAMP-2 shRNA or scramble (control) shRNA, were cultured in nutrient free (without serum and amino acids) Earl`s Balanced Salt Solution (EBSS) or in Dulbecco`s Modified Eagle Medium (DMEM) devoid of serum for 24 hours. Both starvation treatments did not affect the viability of cells as judged by visual inspection and immunoblotting of cleaved caspase-3 (data not shown). Since prolonged starvation under nutrient free conditions induces expression and nuclear translocation of the 


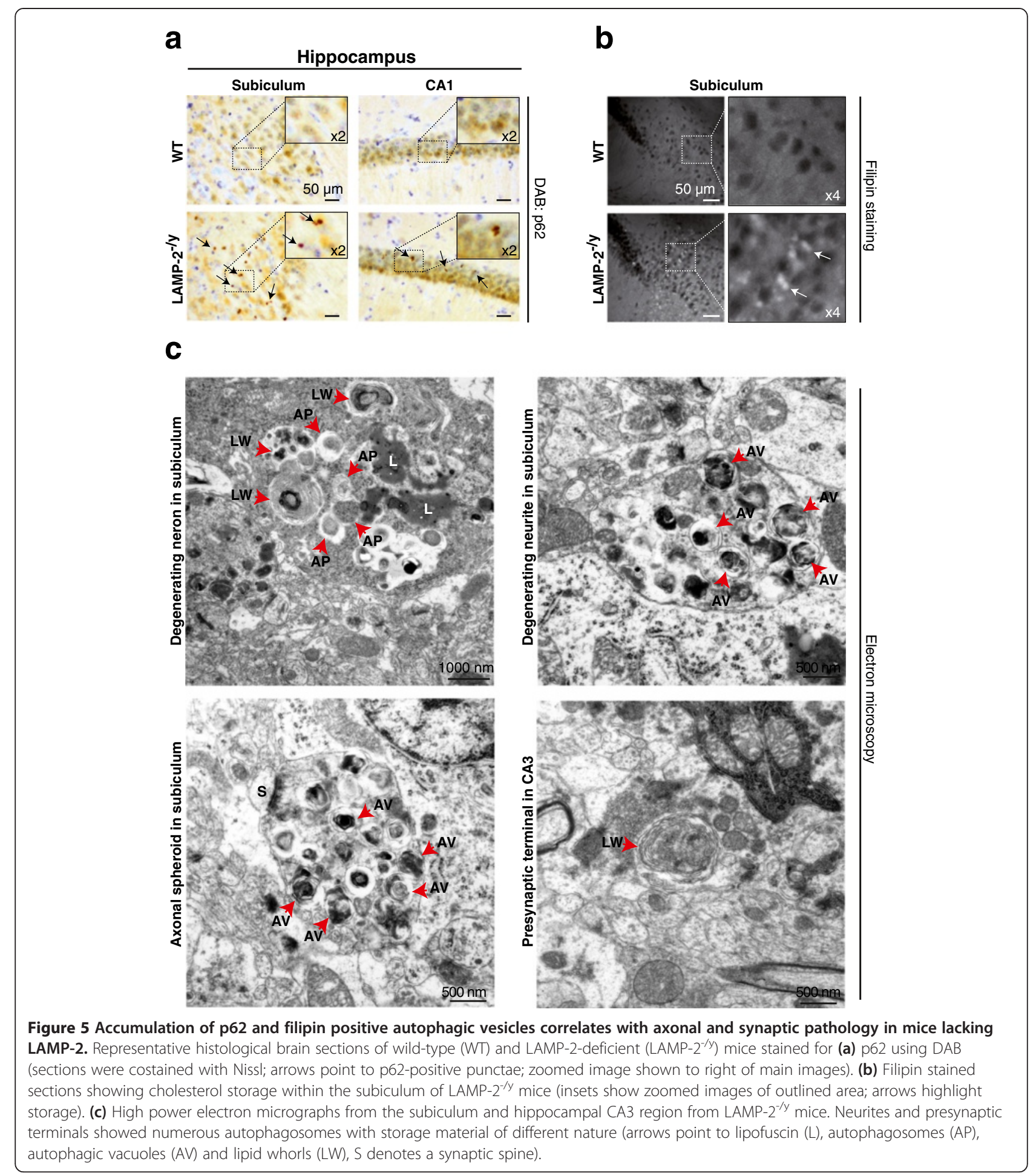

transcription factor EB (TFEB), that is known to modulate lysosomal biogenesis [46], we have assessed protein levels of LAMP-2A, LAMP-2B, LAMP-1 and LIMP-2 via immunoblotting and qRT-PCR analysis. In order to specifically detect LAMP-2A we have used a self-made antibody that only recognizes LAMP-2A as highlighted by immunoblots of HeLa cell extracts overexpressing the three different LAMP-2 isoforms (LAMP-2A, B and C) as well as LAMP-1. Furthermore, no signal was detectable in LAMP-2-deficient brains, confirming the specificity of this antibody (Additional file 7a). Serum removal for 24 hours decreased steady-state levels of MEF2D, 
a

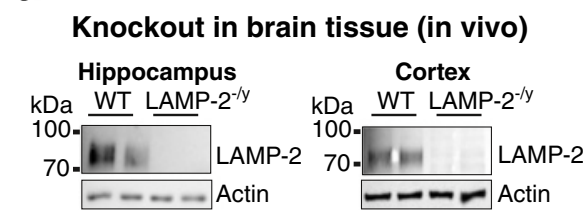

b Hippocampus

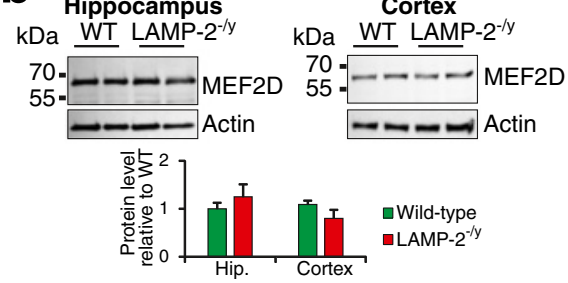

C

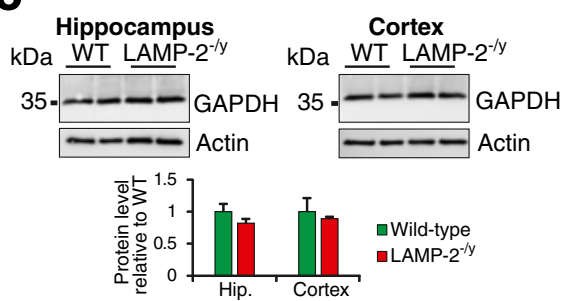

d

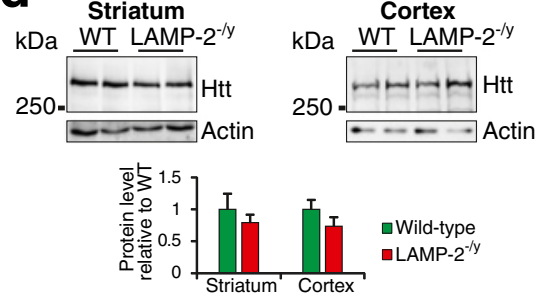

$\mathbf{e}$

Stable knock down in N2a cells (in vitro)

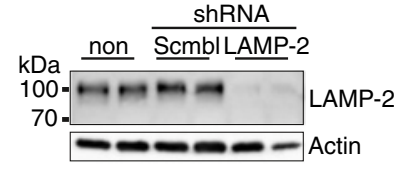

f

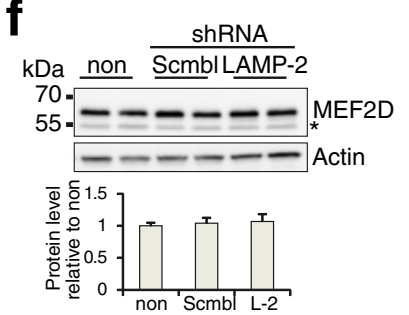

9

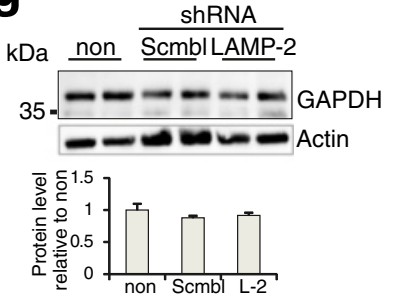

h

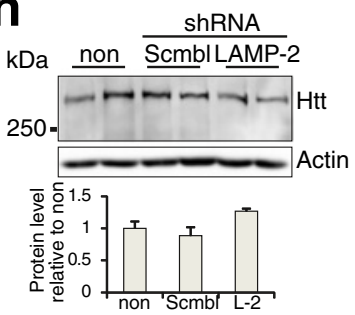

Figure 6 Analysis of steady-state levels of CMA substrates in brain. (a-d) Immunoblots and respective densitometry of lysed hippocampi/ cortices from wild-type (WT) and LAMP-2-deficient (LAMP-2 ${ }^{-1 /}$ ) mice showing protein levels of LAMP-2 (a), MEF2D (b), GAPDH (c) and huntingtin (Htt) (d). (e-h) Immunoblots and respective densitometry of lysates from N2a cells either non-transfected (non) or stably transfected with scramble shRNA (scmbl) or shRNA targeting LAMP-2 mRNA showing steady-state levels of LAMP-2 (e), MEF2D (f), GAPDH (g) or Htt (h) (actin was used to control loading; * denotes a non-specific band for MEF2D). LAMP-2 levels were not affected by scramble (scmbl) shRNA.

independent of the expression of LAMP-2 (Additional file $7 \mathrm{~b})$. Prolonged starvation in complete nutrient-free medium also led to a pronounced decline of MEF2D levels as well as GAPDH (Figure 7a) independent of LAMP-2 expression (Figure 7b). Messenger RNA levels of GAPDH and MEF2D were equal in control and LAMP-2 shRNA starved samples (Additional file 7c).

Under these conditions we observed a significant increase in LAMP-2A expression in control cells as shown by immunoblotting (Figure 7b) and qRT-PCR (Figure 7c). Interestingly, an increase was also observed in LAMP2B (Figure 7c) as well as two other lysosomal membrane proteins investigated namely LAMP-1 (Figure 7d/e) and LIMP-2 (Figure 7f/g).

The knockdown of LAMP-2 did not alter basal (Additional file 7d) or induced MA (Additional file 7e) as indicated by unchanged levels of phosphorylated PRAS40, p62 and LC3-II in control and LAMP-2 shRNA treated N2a cells. Successful induction of MA was monitored by a decline in p62 levels and reduced phosphorylation of PRAS40 (Additional file 7e). Finally, despite the fact that down-regulation of LAMP-2 was reported to lead to an accumulation of another CMA substrate, $\alpha$-syn in cultured neuronal cells $[19,47]$, complete loss of LAMP-2 expression in murine brain did not cause a significant accumulation of either monomeric or high-molecular weight species of $\alpha$-syn within the cortex or hippocampus (Figure 8a/b).

Though prolonged starvation significantly increased LAMP-2A levels in N2a cells, this is not unique to this isoform since a comparable upregulation was also evident for LAMP-2B, LAMP-1 and LIMP-2. Furthermore, 


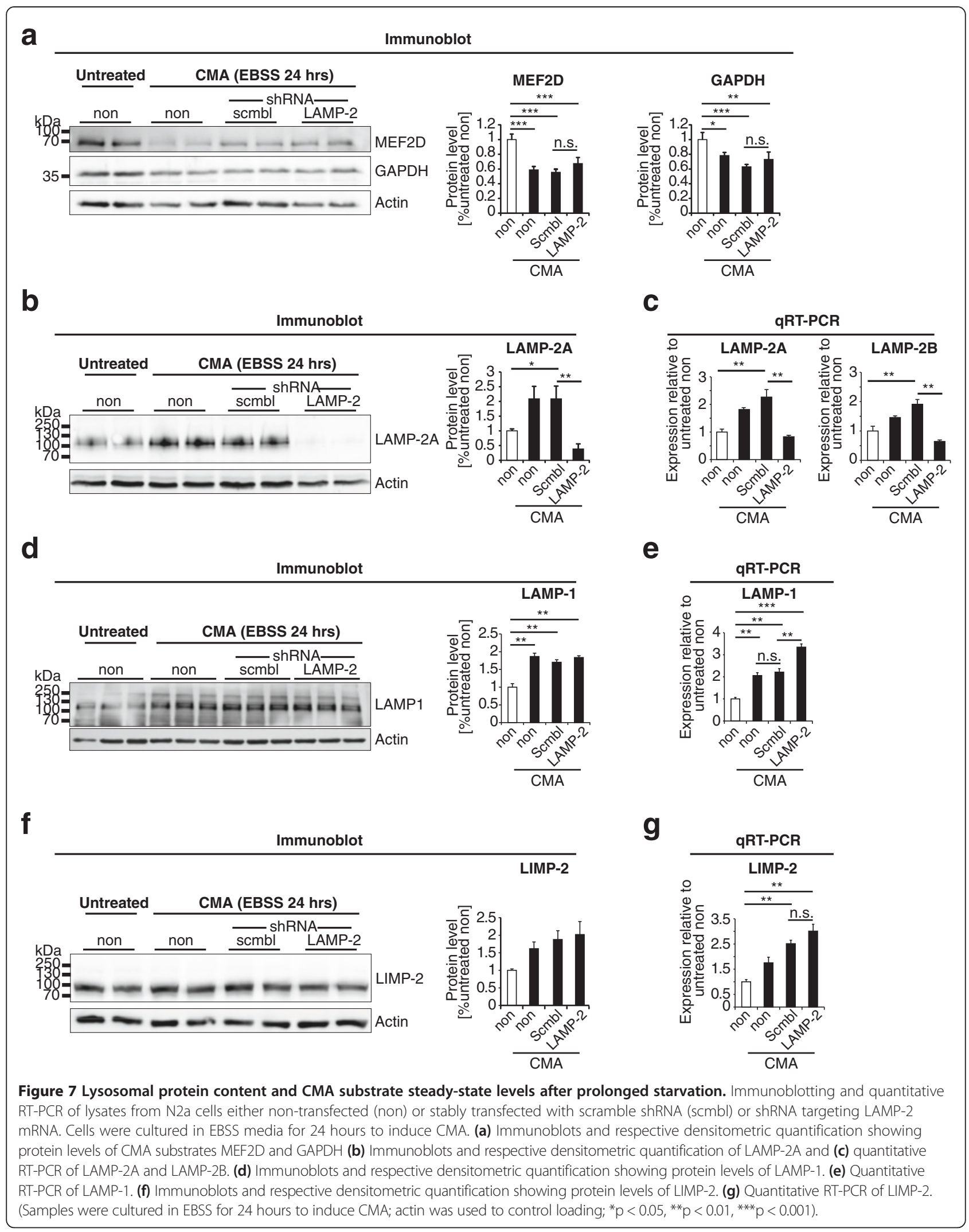




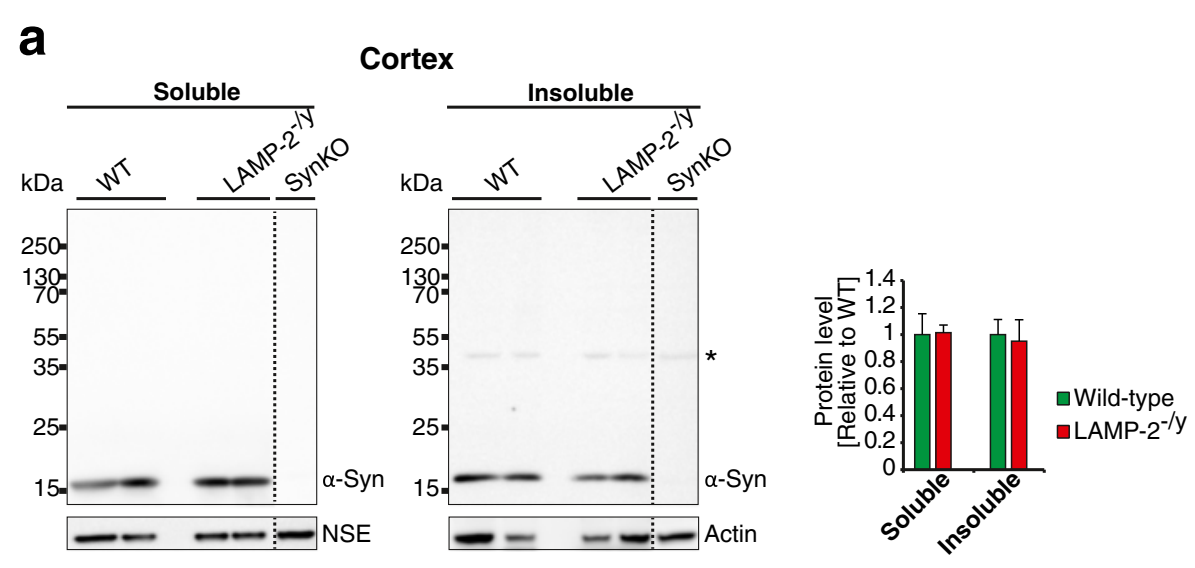

\section{b}

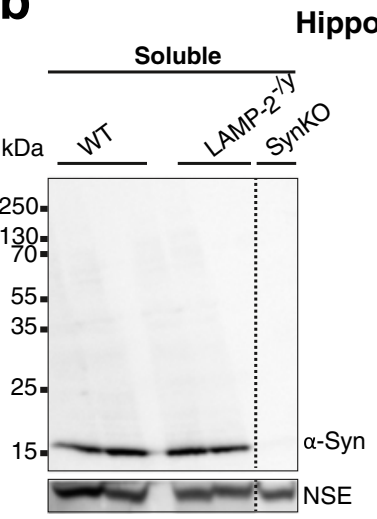

Hippocampus
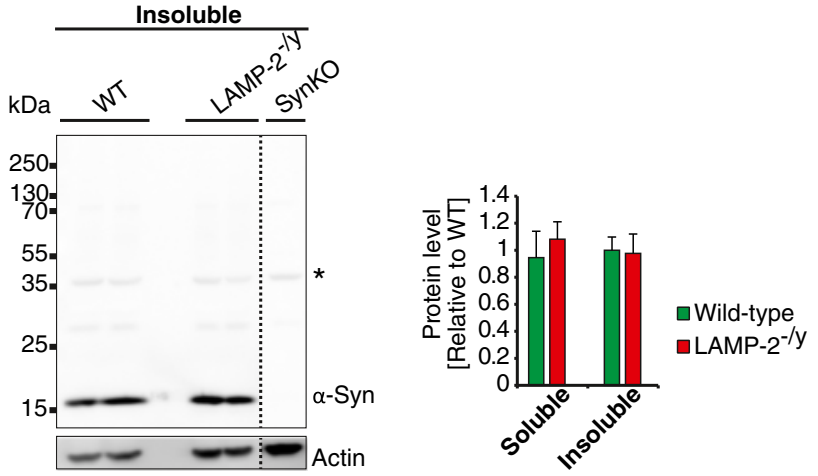

Figure 8 a-synuclein is not accumulated in the absence of LAMP-2 expression. (a/b) Immunoblots and respective densitometric quantification of soluble and insoluble fractions from cortices (a) and hippocampi (b) of LAMP-2-deficient (LAMP-2 $2^{-1 y}$ ) mice and their wild-type (WT) littermates. The asterisk highlights a non-specific band recognized by the a-syn antibody (C-20, Santa Cruz; NSE or actin were used as loading controls).

absence of LAMP-2 did not affect CMA substrate levels in brain tissue and in neuroblastoma cells.

\section{Discussion}

The current report highlights LAMP-2 as a ubiquitously expressed protein of the CNS. In addition to its established role as a receptor for CMA, the function of LAMP-2 in the brain is not yet well defined. Absence of LAMP-2 in brain, especially within the subiculum and CA3 region of the hippocampus, caused pronounced inflammation as well as perturbed lysosomal activity and autophagy indicated by intraneuronal lipid storage, accumulation of autophagic vacuoles and neuronal degeneration in a subpopulation of neurons. Hippocampal neurons are particularly vulnerable to lysosomal storage induced degeneration as highlighted in various mouse models with lysosomal dysfunction $[48,49]$ and in Alzheimer and Parkinson`s disease as well as dementia with Lewy bodies. To some extent this vulnerability can be explained by regional differences in the expression profile of proteins that can induce neurotoxicity under certain pathophysiological conditions such as aggregation of $\alpha$-syn and amyloid- $\beta$. Interestingly, hippocampal but not cortical motor neurons were described as vulnerable to ischemia/reactive oxygen species-induced neurotoxicity through calpain-mediated cleavage of carbonylated hsp70 [50] thereby diminishing its neuroprotective effect [51,52]. A reduction in LAMP-2 expression has been directly linked to low CMA activity as well as increased levels of oxidized proteins in aged livers $[15,45]$ and breast cancer cells [53]. It is therefore tempting to speculate that loss of LAMP-2, especially within hippocampal neurons, leads to an accumulation of oxidized proteins that may contribute to the observed neuropathology. Further studies are necessary in order to elucidate the impact of oxidative stress as a potential cause of CMA blockage on the neuropathology of LAMP-2-deficient mice.

Our data suggest a distinct role of LAMP-2 within the hippocampus that might be causative for the observed intellectual dysfunction in Danon disease patients 
$[25,26,54,55]$. Besides the development of a mild astrogliosis, no other neuropathological changes within the hippocampus were described within the brain of one Danon disease patient [29]. Therefore, the hippocampus should be of special interest when examining brain autopsy material from Danon disease patients in the future. It should also be noted that according to our analyses, LAMP-2A is the most prominent isoform in brain whereas LAMP-2B was only present to a minor degree and LAMP-2C was undetectable under the experimental conditions used. This is in contrast to the described prominent expression of LAMP-2C in murine brain [37]. Therefore, further analysis is necessary in order to elucidate the expression and relative abundance of the different LAMP-2 isoforms in brain.

Due to the rarity of the disease and the limited access to autopsy brain material, LAMP-2-deficient mice provide an important tool in studying the molecular mechanisms underlying Danon disease neuropathology. We observed behavioral changes in these mice which relate to neuromuscular and intellectual dysfunction in patients. Likewise, the neuropathology of LAMP-2-deficient mice and Danon disease patients share common characteristics. Both show pronounced inflammation exemplified by an increased expression of GFAP in activated astrocytes despite absence of obvious signs of pronounced neurodegeneration [29]. Interestingly, GFAP was identified as a modulator of CMA that stabilizes the multimeric CMA translocation complex formed within the lysosomal membrane after CMA induction [56]. Absence of LAMP-2 may interfere with formation of such a complex ultimately leading to the activation of astrocytes.

In addition to inflammation, another prominent feature of the neuropathology described for one Danon disease patient is the presence of electron dense bodies and lipofuscin-rich deposits throughout the brain [29]. In mice, among all other brain regions investigated, the hippocampus displayed most prominent signs of storage especially in the subiculum in which neuronal accumulation of free cholesterol and lipofuscin was evident. Previously, we reported cholesterol storage in LAMP-2-deficient liver [10]. This phenotype was mirrored in murine embryonic fibroblasts lacking LAMP-2 and to a higher extent in cells in which both LAMP proteins were deleted $[9,10]$. Rescue experiments of the observed cholesterol storage by re-expressing both LAMPs into LAMP-1/LAMP-2-double-deficient fibroblasts suggest a distinct role of LAMP-2 but not LAMP-1 in cholesterol metabolism. However, this effect was not isoform specific since all three LAMP-2 variants were equally effective in abolishing the late endosomal/lysosomal cholesterol accumulation [10]. Until now, the precise role of LAMP-2 within this process remains unknown and further analysis is necessary in order to clarify how its expression influences lipid and cholesterol metabolism, especially within neuronal cells.

The pathological hallmark of Danon disease, which was classified as a glycogen storage disorder [25,57], is the presence of accumulated glycogen and autophagic vacuoles within liver and muscle of patients and the mouse model $[4,26,58]$. Therefore, LAMP-2 was suggested to play an important role in the clearance of glycogen and maturation of autophagic vacuoles [5,59]. Interestingly, the glycogen storage disorders Lafora and Pompe disease, caused by mutations within enzymes directly or indirectly involved in glycogen metabolism [57], result in pronounced impairment of autophagy in tissues that accumulated glycogen, including the CNS [60-63]. Therefore, absence of accumulated glycogen within brain samples of LAMP-2-deficient mice may explain the lack of gross abnormalities in neuronal autophagy and suggest a distinct function of LAMP-2 within the hippocampus for autophagic clearance of neuronal proteins.

The most well defined role for LAMP-2A is its function as a receptor for CMA, mediating the lysosomal uptake of selective substrates such as MEF2D [44], Htt $[22,64,65], \alpha$-syn [47] and GAPDH [15]. In our hands, despite apparent unaltered levels of MA and no change in proteasomal activity, loss of LAMP-2 expression did not affect steady-state levels of these proteins in brain lysates or N2a cells under normal conditions. A compensatory upregulation of MA and proteasomal activity that has been found upon blockage of CMA by silencing LAMP-2 expression [66] is therefore unlikely to account for the unchanged levels of CMA substrates in LAMP2-deficient brain. Of note, we also did not observe cytosolic accumulation of MEF2D which has been reported as consequence of CMA blockage by knock-down of LAMP-2 expression [44]. In our hands, neither complete absence nor down-regulation of LAMP-2 affected the typical nuclear localization of this protein further suggesting that LAMP-2 is dispensable for clearance of CMA substrates under the investigated conditions. However, lysosomal uptake experiments of selected CMA substrates would help to clarify the impact of LAMP-2 deficiency within this pathway under normal and stress induced conditions.

With regard to the unchanged levels of $\alpha$-syn in LAMP-2 knockout brains it is interesting to note that elevated expression of $\alpha$-syn in the C57/BL6-N genetic background led to a deterioration of the phenotype of LIMP-2-deficient mice [36]. In contrast, absence of LAMP-2 within the same genetic background did not affect $\alpha$-syn levels suggesting that LAMP-2 is dispensable for its clearance. This correlates with previous studies where double deficiency of LAMP-1 and 2 in mouse embryonic fibroblasts did not affect CMA-mediated 
proteolysis, suggesting existence of alternative receptors or mechanisms for CMA besides LAMP-2A $[9,67]$.

CMA can be induced indirectly by various stimuli such as prolonged starvation or directly by overexpression of its receptor LAMP-2A [68]. Htt, MEF2D, GAPDH and $\alpha$-syn levels were shown by others to be directly linked to expression of LAMP-2A [19,20,44,47]. However, in N2a cells, similar to the situation in LAMP2-deficient brains, a reduction in LAMP-2 expression did not affect steady-state levels of CMA substrates even after prolonged incubation in nutrient or serum-free medium. Furthermore, in control cells, prolonged starvation did not specifically elevate LAMP-2A levels but led to a general increase in lysosomal membrane protein expression namely LAMP-2B, LAMP-1 and LIMP-2. Interestingly, this is in agreement with reports where an increase in LAMP-2A but also LAMP-1 expression was reported after prolonged starvation [15]. Our results are in agreement with the described effect of prolonged starvation that stimulates TFEB induced lysosomal biogenesis and degradation by increasing the expression of lysosomal membrane proteins and hydrolases $[46,69]$. However, further analysis will aid in the interpretation of the role of TFEB in starvation-induced CMA and its effect on expression levels, specifically of LAMP-2A.

\section{Conclusion}

In conclusion, our data suggest a novel role of LAMP-2 for lysosomal and autophagic clearance within hippocampal neurons and contribute to the understanding of neuropathology in Danon disease.

\section{Additional files}

\section{Additional file 1: Neuropathology of LAMP-2-deficient mice.}

Representative histological brain sections from LAMP-2-deficient (LAMP-2 ${ }^{-1 / y}$ ) mice and their wild-type (WT) littermates. (a) Microgliosis observed in LAMP-2 ${ }^{-1 / y}$ animals via CD68 immunological staining (insets depict zoomed images of outlined area, sections were costained with Nissl). Representative histological sections stained with (b) toluidine blue (arrows highlight shrunken cells) and (c) TUNEL (sections were costained with the nuclear stain haematoxylin). (d) Immunoblot of caspase-3 from hippocampal lysates (FL, full length, p17 and p20 refer to caspase-3 cleavage products; NSE was used to control loading).

Additional file 2: No change in LAMP-1 expression in absence of LAMP-2 within brain. (a) Representative histological brain sections stained for LAMP-2 using the DAB method and counterstained with Nissl. No signal was detected in LAMP-2-deficient (LAMP-2 $\left.{ }^{-1 / y}\right)$ brains. Enriched expression was observed in an area of the pons. (b) Immunoblots of lysates from wildtype (WT) and LAMP-2 $2^{-1 y}$ brains probed for LAMP-1 and respective densitometric quantification (actin was used to control loading). (c) Representative histological brain sections stained for LAMP-1 using the DAB method and counterstained with Nissl. Enriched expression was observed in the pyramidal layer of the hippocampus, within the Purkinje cell layer of the cerebellum and within a region of the pons. Zoomed imaged of regions outlined are shown underneath pictures of the hippocampus/pons (\#p>0.05).

Additional file 3: Behavioral analyses of mice at $\mathbf{5 4}$ weeks. (a) LAMP-2deficient (LAMP- $2^{-1 y}$ ) mice showed similar grip strength when compared to wild-type controls. (b) No significant difference in performance using a rotarod was observed between wild-type and LAMP- $2^{-1 y}$ mice. (c) No differences in cage activity were observed between wild-type and LAMP-2 ${ }^{-1 /}$ mice.

Additional file 4: Cathepsin D levels in brain. Densitometric analysis of cathepsin $\mathrm{D}(\mathrm{CtD})$ levels corresponding to representative blots in Figure $4\left(\# p>0.05,{ }^{*} p<0.05,{ }^{* *} p<0.01\right)$.

Additional file 5: Absence of carbohydrate storage or perturbed autophagy in LAMP-2-deficient brain. (a) Representative histological sections from LAMP-2-deficient (LAMP-2 ${ }^{-1 /}$ ) mice and their wild-type (WT) littermates stained with Periodic-Acid-Schiff (PAS). Sections were costained with the nuclear stain haematoxylin. (b) Electron micrograph showing normal morphology within the subiculum of a WT animal. (c) Immunoblots and respective densitometric quantification of hippocampal lysates.

Additional file 6: Lack of LAMP-2 expression does not affect LAMP-1 levels and has no effect on huntingtin or MEF2D. (a) Immunoblot of hippocampal lysates from LAMP-2-deficient (LAMP-2-1y) mice and their wild-type (WT) littermates. (b) Immunoblots of lysates from N2a cells either non-transfected (non) or stably transfected with scramble shRNA (Scmbl) or shRNA targeting LAMP-2 mRNA showing protein levels of LAMP-1. The specificity of the applied shRNA LAMP-2 probe is highlighted by unchanged levels of LAMP-1 protein (actin was used to control loading).

(c) Immunofluorescence of MEF2D (red) and LAMP-2 (green) in primary neurons. (d) Immunofluorescence of MEF2D (red) in hippocampal slices (Dapi (4,6-diamidino-2-phenylindole) was used as a nuclear stain, insets shown zoomed images of area outlined).

Additional file 7: Stable knockdown of LAMP-2 has no effect on expression of MEF2D or GAPDH in N2a cells. (a) Immunoblot of lysates from brain lysates from wild-type (WT) and LAMP-2-deficient $\left(\right.$ LAMP- $\left.2^{-1 /}\right)$ mice as well as HeLa cells overexpressing eGFP, murine LAMP-1 (L1), murine LAMP-2A (L2A), murine LAMP-2B (L2B) or murine LAMP-2C (L2C). The LAMP-2A antibody used in this study is specific for the murine isoform A (actin was used to control loading and LAMP-1 to control for specificity). (b) Immunoblots and respective quanticiation from N2a cells either non-transfected (non) or stably transfected with scramble shRNA (scmbl) or shRNA targeting LAMP-2 mRNA. MEF2D levels decrease after starvation in serum-free DMEM. (c) Quantitative RT-PCR of samples from N2a cells either non-transfected (non) or stably transfected with scmbl shRNA or shRNA targeting LAMP-2 mRNA. MEF2D expression increases after starvation of the cells. However, no significant differences were observed

between scmbl and LAMP-2 shRNA treated samples. GAPDH expression remained constant in all samples ( $\# p>0.05$, ${ }^{* * *} p<0.001$ ). (d) Immunoblot and respective quantification from N2a cells showing lack of changes in macroautophagy upon knockdown of LAMP-2 (p-PRAS40 refers to phosphorylated-PRAS40; actin was used to control loading). (e) Immunoblots and respective densitometric quantification of lysates from N2a cells either untreated or starved for 3 hours to induce macroautophagy.

\section{Abbreviations}

CMA: Chaperone-mediated autophagy; Htt: Huntingtin; a-syn: a-synuclein; MEF2D: Myocyte-specific enhancer factor-2D; GAPDH: Glyceraldehyde-3phosphate dehydrogenase; LAMP-2: Lysosomal associated membrane protein type-2; MA: Macroautophagy.

\section{Competing interests}

The authors declare that they have no competing interests.

\section{Acknowledgements}

This work was supported by the Research Training Group (GRK1459), funded by the Deutsche Forschungsgemeinschaft to J.B., an EU grant to P.S. and J.B. (EU/ALPHA-MAN 261331) and the Interuniversity Attraction Poles Program (IUAP P7/16) of the Belgian Federal Science Policy Office. S.S. received support from the MM Delacroix foundation. F.Z. is supported by the Böhringer Ingelheim Fonds. We thank Meryem Senkara, Inez Götting, Lisa Andresen, Emanuela Szpotovicz and Rasa C. Raithore for excellent technical assistance. 


\section{Author details}

1 Institute of Biochemistry, Christian-Albrechts-Universität zu Kiel, Olshausenstrasse 40, D-24098 Kiel, Germany. ' Laboratory of Biological Psychology, University of Leuven, B-3000 Leuven, Belgium. ${ }^{3}$ Department of Electron Microscopy, Center for Molecular Neurobiology, University Medical Center Hamburg-Eppendorf, D-20246 Hamburg, Germany.

\section{Received: 21 November 2014 Accepted: 30 December 2014} Published online: 31 January 2015

\section{References}

1. Wilke S, Krausze J, Bussow K (2012) Crystal structure of the conserved domain of the DC lysosomal associated membrane protein: implications for the lysosomal glycocalyx. BMC Biol 10:62. doi:10.1186/1741-7007-10-62

2. Neiss WF (1984) A coat of glycoconjugates on the inner surface of the lysosomal membrane in the rat kidney. Histochemistry 80(6):603-608

3. Kundra R, Kornfeld S (1999) Asparagine-linked oligosaccharides protect Lamp-1 and Lamp-2 from intracellular proteolysis. J Biol Chem 274(43):31039-31046

4. Tanaka Y, Guhde G, Suter A, Eskelinen EL, Hartmann D, Lullmann-Rauch R, Janssen PM, Blanz J, von Figura K, Saftig P (2000) Accumulation of autophagic vacuoles and cardiomyopathy in LAMP-2-deficient mice. Nature 406(6798):902-906, doi:10.1038/35022595

5. Eskelinen EL (2006) Roles of LAMP-1 and LAMP-2 in lysosome biogenesis and autophagy. Mol Aspects Med 27(5-6):495-502, doi:10.1016/j. mam.2006.08.005

6. Beertsen W, Willenborg M, Everts V, Zirogianni A, Podschun R, Schroder B, Eskelinen EL, Saftig P (2008) Impaired phagosomal maturation in neutrophils leads to periodontitis in lysosomal-associated membrane protein-2 knockout mice. J Immunol 180(1):475-482

7. Binker MG, Cosen-Binker LI, Terebiznik MR, Mallo GV, McCaw SE, Eskelinen EL, Willenborg M, Brumell JH, Saftig P, Grinstein S, Gray-Owen SD (2007) Arrested maturation of Neisseria-containing phagosomes in the absence of the lysosome-associated membrane proteins, LAMP-1 and LAMP-2. Cell Microbiol 9(9):2153-2166, doi:10.1111/j.1462-5822.2007.00946

8. Huynh KK, Eskelinen EL, Scott CC, Malevanets A, Saftig P, Grinstein S (2007) LAMP proteins are required for fusion of lysosomes with phagosomes. EMBO J 26(2):313-324, doi:10.1038/sj.emboj.7601511

9. Eskelinen EL, Schmidt CK, Neu S, Willenborg M, Fuertes G, Salvador N, Tanaka Y, Lullmann-Rauch R, Hartmann D, Heeren J, von Figura K, Knecht E, Saftig P (2004) Disturbed cholesterol traffic but normal proteolytic function in LAMP-1/LAMP-2 double-deficient fibroblasts. Mol Biol Cell 15(7):3132-3145, doi:10.1091/mbc.E04-02-0103E04-02-0103

10. Schneede A, Schmidt CK, Holtta-Vuori M, Heeren J, Willenborg M, Blanz J, Domanskyy M, Breiden B, Brodesser S, Landgrebe J, Sandhoff K, Ikonen E, Saftig P, Eskelinen EL (2011) Role for LAMP-2 in endosomal cholesterol transport. J Cell Mol Med 15(2):280-295, doi:10.1111/j.1582-4934.2009

11. Konecki DS, Foetisch K, Zimmer KP, Schlotter M, Lichter-Konecki U (1995) An alternatively spliced form of the human lysosome-associated membrane protein-2 gene is expressed in a tissue-specific manner. Biochem Biophys Res Commun 215(2):757-767

12. Hatem CL, Gough NR, Fambrough DM (1995) Multiple mRNAs encode the avian lysosomal membrane protein LAMP-2, resulting in alternative transmembrane and cytoplasmic domains. J Cell Sci 108(Pt 5):2093-2100

13. Lichter-Konecki U, Moter SE, Krawisz BR, Schlotter M, Hipke C, Konecki DS (1999) Expression patterns of murine lysosome-associated membrane protein 2 (Lamp-2) transcripts during morphogenesis. Differentiation 65(1):43-58, doi:10.1046/j.1432-0436.1999.6510043

14. Cuervo AM, Dice JF (1996) A receptor for the selective uptake and degradation of proteins by lysosomes. Science 273(5274):501-503

15. Cuervo AM, Dice JF (2000) Unique properties of lamp2a compared to other lamp2 isoforms. J Cell Sci 113(Pt 24):4441-4450

16. Cuervo AM (2010) Chaperone-mediated autophagy: selectivity pays off. Trends Endocrinol Metab 21(3):142-150, doi:10.003S1043-2760(09)00162-3

17. Zhou D, Li P, Lin Y, Lott JM, Hislop AD, Canaday DH, Brutkiewicz RR, Blum JS (2005) Lamp-2a facilitates MHC class II presentation of cytoplasmic antigens. Immunity 22(5):571-581, doi:10.1016/j.immuni.2005.03.009

18. Valdor R, Mocholi E, Botbol Y, Guerrero-Ros I, Chandra D, Koga H, Gravekamp C, Cuervo AM, Macian F (2014) Chaperone-mediated autophagy regulates T cell responses through targeted degradation of negative regulators of T cell activation. Nat Immunol. doi:10.1038/ni.3003
19. Cuervo AM, Stefanis L, Fredenburg R, Lansbury PT, Sulzer D (2004) Impaired degradation of mutant alpha-synuclein by chaperone-mediated autophagy. Science 305(5688):1292-1295, doi:10.1126/science.1101738305/5688/1292

20. Xilouri M, Brekk OR, Landeck N, Pitychoutis PM, Papasilekas T, Papadopoulou-Daifoti Z, Kirik D, Stefanis L (2013) Boosting chaperonemediated autophagy in vivo mitigates alpha-synuclein-induced neurodegeneration. Brain 136(Pt 7):2130-2146, doi:10.1093/brain/awt131 awt131

21. Koga H, Cuervo AM (2011) Chaperone-mediated autophagy dysfunction in the pathogenesis of neurodegeneration. Neurobiol Dis 43(1):29-37, doi:10.1016/j.nbd.2010.07.006

22. Qi L, Zhang XD, Wu JC, Lin F, Wang J, DiFiglia M, Qin ZH (2012) The role of chaperone-mediated autophagy in huntingtin degradation. PLoS One 7(10):e46834, doi:10.1371/journal.pone.0046834

23. Tobin AJ, Signer ER (2000) Huntington's disease: the challenge for cell biologists. Trends Cell Biol 10(12):531-536

24. Periquet M, Fulga T, Myllykangas L, Schlossmacher MG, Feany MB (2007) Aggregated alpha-synuclein mediates dopaminergic neurotoxicity in vivo. J Neurosci 27(12):3338-3346, doi:10.1523/JNEUROSCI.0285-07.2007

25. Danon MJ, Oh SJ, DiMauro S, Manaligod JR, Eastwood A, Naidu S, Schliselfeld LH (1981) Lysosomal glycogen storage disease with normal acid maltase. Neurology 31(1):51-57

26. Nishino I, Fu J, Tanji K, Yamada T, Shimojo S, Koori T, Mora M, Riggs JE, Oh SJ, Koga Y, Sue CM, Yamamoto A, Murakami N, Shanske S, Byrne E, Bonilla E Nonaka I, DiMauro S, Hirano M (2000) Primary LAMP-2 deficiency causes X-linked vacuolar cardiomyopathy and myopathy (Danon disease). Nature 406(6798):906-910, doi:10.1038/35022604

27. Cheng Z, Fang Q (2012) Danon disease: focusing on heart. J Hum Genet 57(7):407-410, doi:10.1038/jhg.2012.72

28. Stypmann J, Janssen PM, Prestle J, Engelen MA, Kogler H, Lullmann-Rauch $\mathrm{R}$, Eckardt L, von Figura K, Landgrebe J, Mleczko A, Saftig P (2006) LAMP-2 deficient mice show depressed cardiac contractile function without significant changes in calcium handling. Basic Res Cardiol 101(4):281-291, doi:10.1007/s00395-006-0591-6

29. Furuta A, Wakabayashi K, Haratake J, Kikuchi H, Kabuta T, Mori F, Tokonami F, Katsumi Y, Tanioka F, Uchiyama Y, Nishino I, Wada K (2013) Lysosomal storage and advanced senescence in the brain of LAMP-2-deficient Danon disease. Acta Neuropathol 125(3):459-461, doi:10.1007/s00401-012-1075-4

30. Saftig P, Tanaka Y, Lullmann-Rauch R, von Figura K (2001) Disease model: LAMP-2 enlightens Danon disease. Trends Mol Med 7(1):37-39

31. Hsu CY, Uludag H (2012) A simple and rapid nonviral approach to efficiently transfect primary tissue-derived cells using polyethylenimine. Nat Protoc 7(5):935-945, doi:10.1038/nprot.2012.038

32. Naert A, Callaerts-Vegh Z, Moechars D, Meert T, D'Hooge R (2011) Vglut2 haploinsufficiency enhances behavioral sensitivity to MK-801 and amphetamine in mice. Prog Neuropsychopharmacol Biol Psychiatry 35(5):1316-1321, doi:10.1016/j.pnpbp.2011.03.023

33. Blanz J, Stroobants S, Lullmann-Rauch R, Morelle W, Ludemann M, D'Hooge R, Reuterwall H, Michalski JC, Fogh J, Andersson C, Saftig P (2008) Reversal of peripheral and central neural storage and ataxia after recombinant enzyme replacement therapy in alpha-mannosidosis mice. Hum Mol Genet 17(22):3437-3445, doi:10.1093/hmg/ddn237

34. Dobrenis K, Chang HY, Pina-Benabou MH, Woodroffe A, Lee SC, Rozental R, Spray DC, Scemes E (2005) Human and mouse microglia express connexin36, and functional gap junctions are formed between rodent microglia and neurons. J Neurosci Res 82(3):306-315, doi:10.1002/jnr.20650

35. Pfaffl MW (2001) A new mathematical model for relative quantification in real-time RT-PCR. Nucleic Acids Res 29(9):e45

36. Rothaug M, Zunke F, Mazzulli JR, Schweizer M, Altmeppen H, LullmannRauch R, Kallemeijn WW, Gaspar P, Aerts JM, Glatzel M, Saftig P, Krainc D, Schwake M, Blanz J (2014) LIMP-2 expression is critical for betaglucocerebrosidase activity and alpha-synuclein clearance. Proc Natl Acad Sci U S A 111(43):15573-15578, doi:10.1073/pnas.1405700111

37. Fujiwara Y, Furuta A, Kikuchi H, Aizawa S, Hatanaka Y, Konya C, Uchida K, Yoshimura A, Tamai Y, Wada K, Kabuta T (2013) Discovery of a novel type of autophagy targeting RNA. Autophagy 9(3):403-409, doi:10.4161/auto. 2300223002

38. Henell F, Berkenstam A, Ahlberg J, Glaumann H (1987) Degradation of short- and long-lived proteins in perfused liver and in isolated autophagic vacuoles-lysosomes. Exp Mol Pathol 46(1):1-14

39. Komatsu M, Waguri S, Koike M, Sou YS, Ueno T, Hara T, Mizushima N, Iwata J, Ezaki J, Murata S, Hamazaki J, Nishito Y, lemura S, Natsume T, Yanagawa T, 
Uwayama J, Warabi E, Yoshida H, Ishii T, Kobayashi A, Yamamoto M, Yue Z, Uchiyama Y, Kominami E, Tanaka K (2007) Homeostatic levels of p62 control cytoplasmic inclusion body formation in autophagy-deficient mice. Cell 131(6):1149-1163, doi:10.1016/j.cell.2007.10.035

40. Pankiv S, Clausen TH, Lamark T, Brech A, Bruun JA, Outzen H, Overvatn A, Bjorkoy G, Johansen T (2007) p62/SQSTM1 binds directly to Atg8/LC3 to facilitate degradation of ubiquitinated protein aggregates by autophagy. J Biol Chem 282(33):24131-24145, doi:10.1074/jbc.M702824200

41. Jolly RD, Walkley SU (1997) Lysosomal storage diseases of animals: an essay in comparative pathology. Vet Pathol 34(6):527-548

42. Wang L, Harris TE, Lawrence JC Jr (2008) Regulation of proline-rich Akt substrate of $40 \mathrm{kDa}$ (PRAS40) function by mammalian target of rapamycin complex 1 (mTORC1)-mediated phosphorylation. J Biol Chem 283(23):15619-15627, doi:10.1074/jbc.M800723200

43. Sancak Y, Thoreen CC, Peterson TR, Lindquist RA, Kang SA, Spooner E, Carr SA, Sabatini DM (2007) PRAS40 is an insulin-regulated inhibitor of the mTORC1 protein kinase. Mol Cell 25(6):903-915, doi:10.1016/j.molcel. 2007.03.003

44. Yang Q, She H, Gearing M, Colla E, Lee M, Shacka JJ, Mao Z (2009) Regulation of neuronal survival factor MEF2D by chaperone-mediated autophagy. Science 323(5910):124-127, doi:10.1126/science.1166088 323/5910/124

45. Cuervo AM, Wong E (2014) Chaperone-mediated autophagy: roles in disease and aging. Cell Res 24(1):92-104, doi:10.1038/cr.2013.153cr2013153

46. Settembre C, De Cegli R, Mansueto G, Saha PK, Vetrini F, Visvikis O, Huynh T, Carissimo A, Palmer D, Klisch TJ, Wollenberg AC, Di Bernardo D, Chan L, Irazoqui JE, Ballabio A (2013) TFEB controls cellular lipid metabolism through a starvation-induced autoregulatory loop. Nat Cell Biol 15(6):647-658, doi:10.1038/ncb2718

47. Vogiatzi T, Xilouri M, Vekrellis $K$, Stefanis L (2008) Wild type alpha-synuclein is degraded by chaperone-mediated autophagy and macroautophagy in neuronal cells. J Biol Chem 283(35):23542-23556, doi:10.1074/jbc. M801992200

48. Kasper D, Planells-Cases R, Fuhrmann JC, Scheel O, Zeitz O, Ruether K, Schmitt A, Poet M, Steinfeld R, Schweizer M, Kornak U, Jentsch TJ (2005) Loss of the chloride channel ClC-7 leads to lysosomal storage disease and neurodegeneration. EMBO J 24(5):1079-1091, doi:10.1038/sj.emboj.7600576

49. Mitchison HM, Lim MJ, Cooper JD (2004) Selectivity and types of cell death in the neuronal ceroid lipofuscinoses. Brain Pathol 14(1):86-96

50. Zhu H, Yoshimoto T, Imajo-Ohmi S, Dazortsava M, Mathivanan A, Yamashima T (2012) Why are hippocampal CA1 neurons vulnerable but motor cortex neurons resistant to transient ischemia? J Neurochem 120(4):574-585, doi:10.1111/j.1471-4159.2011.07550.x

51. Kirkegaard T, Roth AG, Petersen NH, Mahalka AK, Olsen OD, Moilanen I, Zylicz A, Knudsen J, Sandhoff K, Arenz C, Kinnunen PK, Nylandsted J, Jaattela M (2010) Hsp70 stabilizes lysosomes and reverts Niemann-Pick diseaseassociated lysosomal pathology. Nature 463(7280):549-553, doi:10.1038/ nature08710

52. Nylandsted J, Gyrd-Hansen M, Danielewicz A, Fehrenbacher N, Lademann U, Hoyer-Hansen M, Weber E, Multhoff G, Rohde M, Jaattela M (2004) Heat shock protein 70 promotes cell survival by inhibiting lysosomal membrane permeabilization. The Journal of experimental medicine 200(4):425-435, doi:10.1084/jem.20040531 jem.20040531

53. Saha T (2012) LAMP2A overexpression in breast tumors promotes cancer cell survival via chaperone-mediated autophagy. Autophagy 8(11):1643-1656, doi:10.4161/auto.21654

54. Boucek D, Jirikowic J, Taylor M (2011) Natural history of Danon disease. Genet Med 13(6):563-568, doi:10.1097/GIM.0b013e31820ad795

55. Taylor MR, Ku L, Slavov D, Cavanaugh J, Boucek M, Zhu X, Graw S, Carniel E, Barnes C, Quan D, Prall R, Lovell MA, Mierau G, Ruegg P, Mandava N, Bristow MR, Towbin JA, Mestroni L (2007) Danon disease presenting with dilated cardiomyopathy and a complex phenotype. J Hum Genet 52(10):830-835, doi:10.1007/s10038-007-0184-8

56. Bandyopadhyay U, Sridhar S, Kaushik S, Kiffin R, Cuervo AM (2010) Identification of regulators of chaperone-mediated autophagy. Mol Cell 39(4):535-547, doi:10.1016/j.molcel.2010.08.004S1097-2765(10)00614-3

57. Wolfsdorf Jl, Weinstein DA (2003) Glycogen storage diseases. Rev Endocr Metab Disord 4(1):95-102, doi:5109165

58. Malicdan MC, Noguchi S, Nonaka I, Saftig P, Nishino I (2008) Lysosomal myopathies: an excessive build-up in autophagosomes is too much to handle. Neuromuscul Disord 18(7):521-529, doi:10.1016/j.nmd.2008.04.010 S0960-8966(08)00106-5
59. Eskelinen EL, Illert AL, Tanaka Y, Schwarzmann G, Blanz J, Von Figura K, Saftig P (2002) Role of LAMP-2 in lysosome biogenesis and autophagy. Mol Biol Cell 13(9):3355-3368, doi:10.1091/mbc.E02-02-0114

60. Criado O, Aguado C, Gayarre J, Duran-Trio L, Garcia-Cabrero AM, Vernia S, San Millan B, Heredia M, Roma-Mateo C, Mouron S, Juana-Lopez L, Dominguez M, Navarro C, Serratosa JM, Sanchez M, Sanz P, Bovolenta P, Knecht E, Rodriguez De Cordoba S (2012) Lafora bodies and neurological defects in malin-deficient mice correlate with impaired autophagy. Hum Mol Genet 21(7):1521-1533, doi:10.1093/hmg/ddr590ddr590

61. Puri R, Suzuki T, Yamakawa K, Ganesh S (2012) Dysfunctions in endosomallysosomal and autophagy pathways underlie neuropathology in a mouse model for Lafora disease. Hum Mol Genet 21(1):175-184, doi:10.1093/hmg/ ddr452

62. Valles-Ortega J, Duran J, Garcia-Rocha M, Bosch C, Saez I, Pujadas L, Serafin A, Canas X, Soriano E, Delgado-Garcia JM, Gruart A, Guinovart JJ (2011) Neurodegeneration and functional impairments associated with glycogen synthase accumulation in a mouse model of Lafora disease. EMBO Mol Med 3(11):667-681, doi:10.1002/emmm.201100174

63. Lim JA, Li L, Raben N (2014) Pompe disease: from pathophysiology to therapy and back again. Front Aging Neurosci 6:177, doi:10.3389/fnagi. 2014.00177

64. Thompson LM, Aiken CT, Kaltenbach LS, Agrawal N, Illes K, Khoshnan A, Martinez-Vincente M, Arrasate M, O'Rourke JG, Khashwji H, Lukacsovich T, Zhu YZ, Lau AL, Massey A, Hayden MR, Zeitlin SO, Finkbeiner S, Green KN, LaFerla FM, Bates G, Huang L, Patterson PH, Lo DC, Cuervo AM, Marsh JL, Steffan JS (2009) IKK phosphorylates Huntingtin and targets it for degradation by the proteasome and lysosome. The Journal of cell biology 187(7):1083-1099, doi:10.1083/jcb.200909067

65. Koga H, Martinez-Vicente M, Arias E, Kaushik S, Sulzer D, Cuervo AM (2011) Constitutive upregulation of chaperone-mediated autophagy in Huntington's disease. J Neurosci 31(50):18492-18505, doi:10.1523/JNEUROSCI. 3219-11.2011

66. Massey AC, Kaushik S, Sovak G, Kiffin R, Cuervo AM (2006) Consequences of the selective blockage of chaperone-mediated autophagy. Proc Natl Acad Sci U S A 103 (15):5805-5810. doi:0507436103

67. Wang G, Mao Z (2014) Chaperone-mediated autophagy: roles in neurodegeneration. Transl Neurodegener 3:20, doi:10.1186/2047-9158-3-20

68. Zhang C, Cuervo AM (2008) Restoration of chaperone-mediated autophagy in aging liver improves cellular maintenance and hepatic function. Nature medicine 14(9):959-965, doi:10.1038/nm.1851

69. Sardiello M, Palmieri M, di Ronza A, Medina DL, Valenza M, Gennarino VA, Di Malta C, Donaudy F, Embrione V, Polishchuk RS, Banfi S, Parenti G, Cattaneo E, Ballabio A (2009) A gene network regulating lysosomal biogenesis and function. Science 325(5939):473-477, doi:10.1126/science.1174447

\section{Submit your next manuscript to BioMed Central and take full advantage of:}

- Convenient online submission

- Thorough peer review

- No space constraints or color figure charges

- Immediate publication on acceptance

- Inclusion in PubMed, CAS, Scopus and Google Scholar

- Research which is freely available for redistribution 